\title{
DIAGNÓSTICO DE SAÚDE DAS MULHERES EM IDADE FERTIL DE SERGIPE
}

\author{
Alzira Maria D’avila Nery Guimarães ${ }^{6}$ \\ Sonia Maria Souza ${ }^{7}$ \\ Marta Oliveira Barreto ${ }^{8}$
}

\begin{abstract}
RESUMO:Relato parcial de uma pesquisa realizada pela Secretária de Estado da Saúde em parceria com o UNICEF, intitulado de Diagnóstico Materno-Infantil do Estado de Sergipe. Nesta pesquisa as autoras realizam uma abordagem parcial sobre a saúde das mulheres em idade fértil. Objetivo: Medir por amostragem a nivel estadual os indicadores: estado civil, idade da menarca, início da vida sexual, gravidez, abortos, prevenção do câncer cérvico-uterino e mama, anticoncepção e utilização dos serviços de saúde. Os resultados foram coletados através da aplicação de um formulário estruturado, abrangendo variáveis préconcepcionais, biológicas e sociais. Os dados obtidos fornecem subsídios para ampla discussão sobre a saúde das mulheres em Sergipe.
\end{abstract}

UNITERMOS: Saúde Reprodutiva - Mulher - Planejamento Familiar

\section{INTRODUÇÃO}

Esta pesquisa é parte de um Diagnóstico de Saúde Materno-Infantil realizada pela Secretaria de Estado da Saúde de Sergipe com promoção do UNICEF, no ano de 1994. Participaram desta pesquisa 20 profissionais da saúde, de nivel superior, selecionados para tal fim, sob a coordenação da autora deste trabalho que representava a Secretaria de Estado da Saúde, João Alberto Cardoso Siqueira, pediatra que representou a UNICEF em Sergipe e do Coordenador de Saúde do UNICEF para Sergipe e Bahia: Dr. Mário Glisser. Considerando a amplitude da pesquisa que trabalhou com inúmeros indicadores, apresentaremos neste trabalho apenas uma parte da mesma referente ao Diagnóstico de Saúde das Mulheres em Idade Fértil de Sergipe.

Os diagnósticos de Saúde na Comunidade devem ser encarados não como atividades isoladas, mas sim dentro de um contexto abrangente de utilização permanente de informações epidemiológicas para avaliação e planejamento dos Serviços de Saúde.

Sergipe, como a maioria dos Estados brasileiros, não dispõe de informações confiáveis a respeito de indicadores básicos fundamentais levando em consideração informações com base populacional. Essa carência de

\footnotetext{
6 Enfermeira. Especialista em Saúde Pública e Professora Auxiliar do Dept. de Enfermagem e Nutrição da UFSE.

7 Enfermeira. Especialista em Saúde Pública e Administração Hospitalar Coordenadora do Programa Materno-Infantil da SES-Sergipe.

${ }^{8}$ Enfermeira. Especialista em Saúde Pública. Diretora Central de Saúde da SES-Sergipe
} 
informações torna quase impossivel o planejamento de ações de saúde voltadas para a mulher. Além de serem instrumentos importantíssimos para um planejamento realista, os indicadores servem também para posterior avaliação do impacto dos programas a serem implantados através da repetição de pesquisa após um determinado intervalo de tempo.

A presente pesquisa teve como objetivo medir por amostragem a nivel estadual os seguintes indicadores de Saúde, para mulheres em idade fértil (15 a 49 anos).

- Estado Civil

- Filhos tidos

- Idade da menarca

- Início da vida sexual

- Gravidez

- Abortos

- Prevenção do câncer de colo de útero e mama

- Anticoncepção

\section{METODOLOGIA}

A fim de atingir os objetivos propostos neste trabalho, seguiram-se várias etapas, assim especificadas: Variáveis do estudo, População, Amostragem, Instrumentos da pesquisa, Coleta de dados, Processamento e Análise dos dados.

Sob o ponto de vista da metodologia utilizada, diagnóstico de Saúde das mulheres em Sergipe, foi realizada através do estudo das seguintes variáveis:

Variáveis pré-concepcionais biológicas: Idade da menarca, Iniciação sexual, Gestações, Número de partos, Idade do primeiro filho, Idade do último filho, Cesárias, Abortamentos, Anticoncepção.

Variáveis pré-concepcionais sociais: Raça, Estado civil, Acesso aos serviços de saúde.

Considerando os indicadores a serem avaliados, estudou-se uma amostra de cerca de 2100 mulheres em idade fértil (15 a 49 anos) em todo o Estado, pois permitiria obter um margem de erro inferior a $5 \%$ para a maioria dos indicadores.

\section{A) AMOSTRAGEM}

O objetivo do processo de amostragem foi obter uma amostra representativa das mulheres nas faixas etárias estudadas residentes no Estado de Sergipe. O processo de amostragem escolhido foi o de estágios múltiplos, divididos em três etapas. Na primeira, sorteou-se os municípios a serem pesquisados; na segunda os setores censitários dentro de cada municipio e na terceira etapa sorteou-se o ponto inicial dentro de cada setor, a partir do qual foram visitados um certo número de municípios.

Foram sorteados 20 conglomerados em todo o Estado, cada um com 8 setores censitários, assim distribuídos: $06 \mathrm{em}$ Aracaju, pois a capital detém aproximadamente $30 \%$ da população estadual, e 14 no interior. Os municipios 
foram listados por região de saúde a que pertenciam e com população acumulada, o que permitiu o sorteio com probabilidade proporcional ao tamanho, garantindo uma ampla representatividade geográfica da amostra.

Em cada município foram sorteados oito setores censitários definidos pelo IBGE(48 em Aracaju e 112 no interior), cada qual com 200 a 300 domicílios. Dois dos 20 conglomerados foram sorteados em municípios com menos de 8 setores censitários; assim, cada setor foi sorteado uma vez e a seguir sorteado novamente o número de setores que faltou para completar 8 . Após o sorteio dos setores censitários, sorteava-se o ponto inicial a partir do qual eram identificados domicílios a serem visitados até serem encontrados 13 mulheres em idade fértil ( 15 a 49 anos de idade), residentes nestes domicílios, que constituíram a amostra do estudo.

Os municipios incluidos foram os seguintes: Aracaju, Carira, Carmópolis, Cedro de São João, Estância, Itabaiana, Itaporanga, Lagarto, Nossa Senhora da Glória, N.S. do Socorro, Pedrinhas, Santana do São Francisco, $\mathrm{St}^{\circ}$ Amaro das Brotas e Simão Dias.

Sergipe possui 75 municipios; todos eles foram contactados previamente e deram apoio logístico à pesquisa, o que incluia principalmente a presença de guias locais. Toda a equipe envolvida com a pesquisa, em um total de 20 profissionais ( 2 coordenadores estaduais, 4 supervisores, 12 entrevistados e 02 analistas de sistemas), participou de um treinamento teórico/prático de 40 horas a fim de ser capacitada para a realização da pesquisa. Para deslocamento, foram utilizados 4 veículos que ficaram inteiramente à disposição das equipes durante a realização da pesquisa.

\section{B) INSTRUMENTO DA PESQUISA}

Utilizou-se como instrumento da pesquisa um formulário estruturado para orientação da entrevista, abrangendo as variáveis pré-concepcionais, biológicas e sociais.

Este formulário foi aplicado à amostra, que envolvia todas as mulheres de 15 a 49 anos de idade encontradas nos domicilios visitados, o que permitiu aferir os indicadores propostos. O documento utilizado para a realização da entrevista encontra-se no apêndice.

\section{C) COLETA DE DADOS}

Os dados forma coletados entrevistando-se individualmente todas as mulheres de 15 a 49 anos de idade encontradas nos domicilios visitados.

\section{D) PROCESSAMENTO E ANÁLISE DOS DADOS}

Todo o processamento, ou seja codificação, digitação, limpeza e edição dos dados, foi realizado no setor de processamento de dados da Secretaria de Estado da Saúde, sob orientação de 02 Analistas de Sistemas. 


\section{RESULTADOS E DISCUSSÕES}

\section{- Características Demográficas}

Nesta pesquisa foram entrevistadas 2201 mulheres na faixa etária de 15 a 49 anos de idade, sendo $31,9 \%$ residentes na capital, $36,7 \%$ no interior urbano e $31,4 \%$ na área rural.

$\mathrm{Na}$ Tabela I pode ser observado que $15,1 \%$ do total das mulheres inquiridas eram adolescentes e $54,6 \%$ estavam distribuidas na faixa de 20 a 34 anos de idade. Com relação à raça, $24,3 \%$ das entrevistadas eram brancas, $10,9 \%$ negras e $64,8 \%$ pardas.

Em estudo comparativo deste diagnóstico com o Censo Demográfico realizado no ano de 1991, podemos verificar que a população de mulheres residentes na área urbana coincide com o percentual encontrado nesta pesquisa(68,6\%). Fazendo a análise comparativa dos dados relativos à raça com os obtidos no PNAD(Pesquisa Nacional de Amostra nos Domicilios) ano 1987, podemos constatar que a distribuição das mulheres brancas, negras e pardas aproxima-se dos percentuais encontrados nesta pesquisa.

No que se refere ao estado civil, $64,0 \%$ das mulheres da capital e $71,1 \%$ no interior do Estado viviam em situação marital no período da realização da pesquisa.

Quanto ao número de filhos por domicilios, foi obtida a média de 1,7 filhos para a Capital e 2,1 para o Estado, enquanto que o número de moradores por domicilio corresponde a uma média de 3,6 (Tabela II)

\section{- História Reprodutiva}

Podemos verificar que a primeira menstruação(menarca) ocorreu majoritariamente na faixa dos 13 a 14 anos, tanto na Capital como no interior do Estado (zona urbana e rural), 45,3\% e 45,2\% respectivamente.

Quanto à iniciação da atividade sexual, foi observado que esta ocorreu com maior freqüência entre 16 e 19 anos de idade, tanto na Capital como no interior, havendo, no entanto, uma diferença significativa na faixa de menores de 16 anos de idade, onde o interior apresentou uma precocidade maior na idade da primeira relação sexual(13,9\% capital, 23,0\% interior urbano e $27,8 \%$ interior rural).

Com relação à idade do primeiro parto, 38,4\% das mulheres da capital, $50,9 \%$ do interior urbano e $55,3 \%$ da área rural tiveram filhos antes dos 20 anos de idade, ficando com uma média de 21,4 anos para a capital e 19,8 para o interior.

Quanto à idade do último parto, houve um equilíbrio tanto na capital como no interior, sendo obtida um média de 26,7 anos. É importante ressaltar que uma de cada doze mulheres teve filhos antes dos 16 anos de idade.

A média de filhos vivos na capital foi de 1,82 e no interior 2,69(zona urbana e rural). No que diz respeito ao número de filhos vivos, aproximadamente $35,0 \%$ das mulheres, na Capital, tinham 3 ou mais filhos 
vivos, contra o interior urbano com $46,0 \%$ é a área rural $51,0 \%$, havendo uma diferença significativa quando comparado o interior com a Capital.

No que tange ao numero de filhos mortos, cerca de 7,6 das mulheres do Estado já haviam tido um ou mais filhos mortos, sendo ligeiramente superior nas mulheres do interior, principalmente as residentes na zona rural. Esta tabela evidencia que $40,4 \%$ das mulheres da capital e $24,2 \%$ do interior já haviam tido pelo menos um filho por cesariana, sendo a proporção muito maior nas mulheres da Capital.

Das $28,7 \%$ das mulheres da Capital e aproximadamente $31,0 \%$ das do interior já haviam tido algum tipo de aborto, sendo que grande parte dos abortos declarados foram expontâneos. Este indice é considerado extremamente elevado, especialmente no interior do Estado. Este fato pode ser explicado provavelmente pela dificuldade que a mulher encontra em referir a realização do aborto provocado, fato considerado ilegal no Brasil. Quanto ao número de abortos tanto nos provocados como nos expontâneos, houve um equilibrio nos índices na Capital e no interior do Estado.

Ainda pode ser observada a distribuição dos métodos utilizados nos abortos provocados, sendo mais freqüente o uso do Misoprostol(CITOTEC), com 48,3\% na Capital, $59,1 \%$ das mulheres utilizaram este método, contra $34,8 \%$ do interior urbano e $56,0 \%$ da área rural, chamando atenção este último que apresentou índice aproximadamente igual as mulheres da capital. Outro método utilizado com grande freqüência foram os chás e as ervas que obtiveram um percentual de $15,9 \%$ na Capital, $46,8 \%$ no interior urbano e $32,0 \%$ na área rural, com uma proporção quase 3 vezes maior no interior urbano que na Capital.

Quando foram inquiridas sobre a realização do aborto, 67,3\% das mulheres da capital referiram que haviam feito sozinhas, contra $80 \%$ do interior. A participação de um médico também foi mais freqüente na Capital, com um percentual de $20,0 \%$. Ainda em relação a quem realizou o aborto, a participação da "Curiosa" destacou-se na capital do Estado com uma freqüência três vezes maior que no interior urbano. Não foi referida a participação da "curiosa" na realização do aborto no interior rural.

Das mulheres que fizeram o aborto, $28,1 \%$ informaram ter sido hospitalizadas por complicações em decorrência do mesmo, totalizando uma média de 2,6 dias de internação para as mulheres da Capital e 4,3 para as do interior do Estado.

Quanto aos serviços de saúde, observou-se que $48,8 \%$ das mulheres fizeram consulta ginecológica nos últimos 12 meses, sendo 60,2\% na Capital e $43,0 \%$ no interior; outrossim, as mulheres do interior em uma maior proporção procuraram consulta apenas por gravidez(32\%). Das mulheres que consultaram por motivo ginecológico $36,4 \%$ informaram ter realizado a consulta por estarem com leucorréia e $34,8 \%$ com dor pélvica.

Cerca de $60,7 \%$ das mulheres referiram haver realizado alguma vez o exame preventivo de câncer uterino( $64,7 \%$ e $58,4 \%$ capital e o interior respectivamente). Das mulheres que fizeram o exame preventivo, $61,8 \%$ da Capital, $58,2 \%$ do interior urbano e $51,6 \%$ do interior rural afirmaram que 
fizeram o exame no último ano, sem diferença significativa entre Capital e interior. Estes dados refletem que o acesso está quase igual na Capital e no interior e pode ser explicado através da realização do Programa Pró-Mulher em todo o Estado, nos últimos três anos.

Em relação ao exame de mama, apenas 30,3\% das mulheres da Capital, $17,5 \%$ interior urbano e $10,9 \%$ interior rural referiram que já realizaram este exame. Destas mulheres, 5,7\% afirmaram ter feito o exame por doença e $19,5 \%$ como exame preventivo do câncer. Estes exames são de fundamental importância, considerando a iniciação precoce das atividades sexuais, por parte das mulheres, e devido à alta incidência do câncer de útero e mama.

Foi evidenciada a utilização de métodos contraceptivos, mostrando que $49,5 \%$ das mulheres na Capital e $51,6 \%$ do interior já utilizaram algum método anticonceptivo no mês anterior à entrevista, tendo a pílula predominado na Capital com $37,1 \%$ e a laqueadura no interior $46,5 \%$. Chama a atenção a presença de $3,8 \%$ da vasectomia, com variação ligeiramente discreta entre a Capital e o interior, e apenas $0,3 \%$ de DIU, método que deveria receber maior prioridade, por razões econômicas, de continuidade de uso e de seu caráter de reversibilidade. Ainda com relação à laqueadura, aproximadamente $20 \%$ das mulheres laqueadas no interior do Estado o fizeram antes dos 25 anos de idade.

Quando foram inquiridas quem havia orientado sobre o método, $53,4 \%$ das mulheres na capital e $40,3 \%$ do interior afirmaram ter recebido orientação médica. Destaca-se entretanto, a utilização por conta própria, com um percentual de $43,5 \%$.

Quando perguntadas se foi ensinado o uso do método, $94,2 \%$ das mulheres que não utilizaram o método por conta própria informaram que foram orientadas quanto á utilização do mesmo.

Em relação ao local de aquisição do mesmo, 75,3\% das mulheres da Capital informaram que obtiveram o método na farmácia, contra quase $91,0 \%$ do interior. Estes dados refletem a necessidade premente da implantação de uma política de planejamento familiar na rede de serviços básicos da saúde. Ainda nessa Tabela pode ser observado que $90,9 \%$ das mulheres apresentavam-se satisfeitas com o método que estavam utilizando.

Foi possível verificar que $17,2 \%$ do número total de mulheres inquiridas na Capital estavam laqueadas, enquanto que no interior urbano $26,2 \%$ e rural $21,7 \%$.

No que tange à idade ao laquear, $52,4 \%$ das mulheres haviam sido submetidas à laqueadura tubária antes dos 30 anos de idade, sendo que $1,8 \%$ a realizaram ainda na adolescência. Das mulheres laqueadas, $21,6 \%$ tinham um ou dois filhos. Este percentual foi maior na Capital 28,1\%, contra $19,1 \%$ no interior, aproximadamente $1 / 4$ das mulheres da Capital; $11,8 \%$ das do interior urbano e $6,1 \%$ das da área rural pagaram pela laqueadura. Este procedimento foi realizado predominantemente por ocasião da cesariana, $53,7 \%$ na Capital e $43,6 \%$ no interior, sendo que a cirurgia específica foi mais freqüente no interior(urbano e rural).

A decisão da laqueadura coube predominantemente ao casal em Aracaju, $52,1 \%$, seguidos da decisão por conta própria, $33,2 \%$. No interior ocorreu o 
inverso, a decisão por conta própria destacou-se com $42,6 \%$, enquanto que a decisão atribuida ao casal ficou com cerca de 34,1\%. A decisão recomendada pelo médico obteve um percentual maior no interior(urbano e rural) $19,6 \%$ e $23,7 \%$ respectivamente do que na Capital $13,2 \%$.

\section{CONCLUSÕES E RECOMENDAÇÕES}

- Algumas características referentes à vida reprodutiva expõem as mulheres de Sergipe a uma série de agravos à sua saúde e de seus filhos.

A iniciação precoce da vida sexual, a freqüente ocorrência de gestações na adolescência e a alta proporção de abortamentos com conseqüentes complicações são motivos de preocupação. Praticamente uma em cada três mulheres já havia tido pelo menos um aborto, principalmente da forma expontânea. A maioria dos abortos induzidos foi realizada por conta própria, através do medicamento Misoprostol. Uma grande parte apresentou problema de saúde e precisou de hospitalização.

- O acesso aos serviços de saúde reflete, além da baixa procura, desigualdade entre as mulheres da Capital e interior.

Pouco menos da metade das mulheres havia consultado um ginecologista nos últimos doze meses. Das que procuraram, 1/4 estava grávida, sendo que as mulheres da Capital são mais servidas que as do interior. A realização do exame preventivo para o câncer de útero cobriu quase $2 / 3$ das mulheres estudadas sem grande diferença entre Capital e interior. A leucorréia e dor pélvica foram citadas como principais causas da consulta ginecológica.

- A utilização de métodos contraceptivos merece amplo debate.

Cerca da metade das mulheres estudadas havia utilizado algum método anticoncepcional no último mês, $4 \%$ estavam grávidas e $2,8 \%$ queriam engravidar. De todas as mulheres, aproximadamente $22 \%$ estavam laqueadas e $17 \%$ faziam uso de anticoncepcionais orais. Os métodos foram orientados em mais de $40 \%$ das vezes por médicos, acompanhados da mesma proporção por conta própria. Chama atenção a pouca utilização do DIU e a presença de 3,8\% de vasectomia.

- A falta de uma política efetiva de planejamento familiar tem deixado como únicas alternativas a laqueadura tubária e os anticoncepcionais orais.

A pilula anticoncepcional predominou na Capital, sendo o segundo método de escolha no interior. É importante ressaltar que o uso de anticoncepcional oral sem a devida orientação e o conhecimento dos fatores de risco associados pode ser extremamente prejudicial à saúde da mulher.

Quanto à laqueadura, $20 \%$ das mulheres com idade inferior a 25 anos de idade estavam laqueadas. As conseqüências psicossociais e físicas da laqueadura merecem estudos adicionais.

- Os dados obtidos nesta pesquisa fornecem subsídios para ampla discussão sobre as condições das mulheres de Sergipe.

$\dot{E}$ inegável reconhecer que existe uma necessidade de intensificação de uma política de saúde que viabilize a implantação do PAISM, definindo prioridades e estratégias de acordo com informações com base populacional. 


\section{REFERÊNCIAS BIBLIOGRÁFICAS}

1. BARROS, Fernando C; VICTORA, Cezar G. Epidemiológica da Saúde Infantil. $2^{\mathrm{a}}$ ed. São Paulo: HuCITEC/UNICEF. 1994

2. FONTES, José Américo Silva. Assistências Materno Infantil: Privilégios de Poucos, Direitos de Todos. Rio de Janeiro: Cultura Médica, 1984

3. FUNDAÇÂO INSTITUTO BRASILEIRO DE GEOGRAFIA E ESTATISTICA. Pesquisa Nacional por Amostra de Domicílios.(PNAD). Rio de Janeiro: FIBGE. 1987

4. MINISTÉRIO DA SAUUDE. Normas de Atenção Integral á Saúde do Adolescente - v. I, II , III. Brasilia: COSMI/MS. 1993

5 Manual de Normas para o Controle do Câncer Cérvico Uterino e Mama. Brasilia: DINSAMI/MS. 1984

6. Assistência Integral á Saúde da Mulher - Textos Básicos de Saúde. Brasília: DNDCD/MS. 1989

7. Assistência Pré Natal. Normas e Manuais Técnicos. Brasilia. DINSAMI/MS. 1986

8. SECRETARIA DE ESTADO DE SAUUDE DA BAHIA. A Saúde das Crianças e Mulheres da Bahia. SESAB/UNICEF. 1992

9. SECRETARIA DE ESTADO DA SAUDE DE SERGIPE. Crianças e Adolescentes em Sergipe - A Saúde, A Educação, O Trabalho. Governo de Sergipe/UNICEF. 1991. 